\title{
New methods and equipment for three-dimensional laser scanning, mapping and profiling underground mine cavities
}

\author{
SN Ahmed Clickmox Solutions Inc., Canada \\ JD Gagnon Clickmox Solutions Inc., Canada \\ MN Makhdoom Clickmox Solutions Inc., Canada \\ R Naeem Clickmox Solutions Inc., Canada \\ J Wang Clickmox Solutions Inc., Canada
}

\begin{abstract}
In this paper, methods related to 3D laser scanning and profiling underground mine cavities that are radically different from traditional cavity monitoring systems are presented. Traditional systems suffer from many drawbacks, including long scan time, being limited to stationary scan capability requiring point cloud stitching, long set up time, bulky systems and low point cloud density. V-SCAN3D offers a highly versatile scanning system that is fast, capable of performing both mobile and stationary scans, and is lightweight and compact. Simultaneous localisation and mapping (SLAM) software enables mobile scanning and generation of $3 D$ maps. This paper discusses case studies related to their use in real mining environments and how it dramatically increases safety and scanning efficiency.
\end{abstract}

Keywords: cavity monitoring systems, cavity surveying, underground mine scanning

\section{Introduction}

Cavity surveys are routinely performed in underground mines for various reasons, including excavated rock mass measurements, monitoring of cavity geometry and its geological features, and identification of potentially dangerous rock mass conditions. These cavities include stopes, mine drifts, ore passes and other underground openings.

Most mines use cavity monitoring systems (CMS) for this purpose. These systems are composed of a single laser, which is continuously rotated at two angles to measure distances of obstructions around the laser head (Teledyne Optech Incorporated 2017). In general, time-of-flight measurements are performed where the return time of the laser pulse is measured with reference to a precise trigger (Amman et al. 2001). The distance is then calculated by multiplying this time with the velocity of light. Half of this distance gives the distance of the obstruction from the laser source. All such points collected during the scan are collectively called a point cloud. This point cloud is used to create a wire mesh of the cavity surface, which can then be used to create a solid surface, and thus calculate the volume of the cavity.

An important part of this process is to register the CMS with respect to the mine coordinate system. This is generally done before the scanning is performed, through a process that involves mounting prisms at previously surveyed points. Once the system has been registered, the scanning process is started, which, for most instruments, takes between six and 10 minutes. It is worth mentioning that to effectively scan the cavity, the system must be inserted into the cavity and held stationary at a point where the possibility of shadows is minimised. This is done by mounting the scanner head on a boom and pushing the boom into the cavity. 
Since a typical CMS scanning head with battery weighs over $10 \mathrm{~kg}$, the process is seldom performed by one person, and generally requires two or more surveyors working together. In terms of effectiveness, ease of use and total scanning time, this method has the following drawbacks.

- The complete scanning process, including registration, may take an hour or more.

- The system may miss portions of the cavity, due to obstructions.

- The system is heavy and therefore requires more than one person to perform the survey.

- Point cloud density is low.

- The system must be kept stationary during scans.

In this paper, a new light weight and compact 3D laser scanning system that overcomes the drawbacks of traditional CMS is proposed.

\section{$2 \quad$ A new approach to cavity scanning}

A new methodology and system to perform underground mine cavity scanning has been developed. It overcomes the drawbacks mentioned earlier and allows for more accurate, faster and cost effective monitoring of mine cavities. This new method involves a system that is light enough to be mounted on aerial drones and is capable of performing both stationary and mobile scans. The mobile scanning capability allows the system to scan areas that are blocked by obstructions in traditional CMS. This also significantly reduces the set up and scanning time.

In this system, the intensity of reflected light is also measured, allowing for the deduction of geological features, such as cracks and faults, from the data gathered. Furthermore, reflected surfaces on surveyed points can be used to register the point cloud during post-processing, thus eliminating the need to perform the usual time-consuming pre-scan registration.

\section{System description}

The V-SCAN3D system consists of a 2D scanning laser head on a rotating platform (Figure 1). A mirror in the scanning laser head rotates to allow scanning at a frequency of $40 \mathrm{~Hz}$. The laser head itself is rotated at different frequencies depending on the mode of scanning.

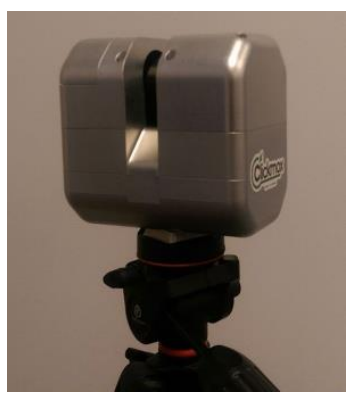

Figure 1 V-SCAN3D

The system works on the time-of-flight measurement principle described earlier in this paper, and in addition, measures the intensity of the reflected light.

The system is capable of performing both stationary and mobile scanning. Mobile scanning is accomplished through a simultaneous localisation and mapping (SLAM) algorithm, which allows feature-rich underground mine cavities to be mapped with the system on a mobile platform, such as an aerial drone. The plastic enclosure version of V-SCAN3D weighs only $650 \mathrm{~g}$ and can therefore be mounted on mid-sized aerial drones suitable for underground mines. 
For stationary scans, the system takes less than 40 seconds to perform a complete scan. This should be compared with the minimum six minute scan time with the traditional CMS. A complete 40 second scan by V-SCAN3D captures more than 1.7 million points. This should be compared with the less than 60,000 points captured by traditional CMS equipment in one six-minute scan (Teledyne Optech Incorporated 2017).

\section{$4 \quad$ Tests and results}

A number of tests in the lab and mine environments were performed to understand the effectiveness and limitations of the system. The results of some of these stationary and mobile scanning tests are presented here.

\subsection{Stationary scanning}

In order to measure accuracy, precision and resolution, $51 \mathrm{~mm}$ wide reflective tapes on a board separated by 40,15 and $5 \mathrm{~mm}$ were used (Figure 2).

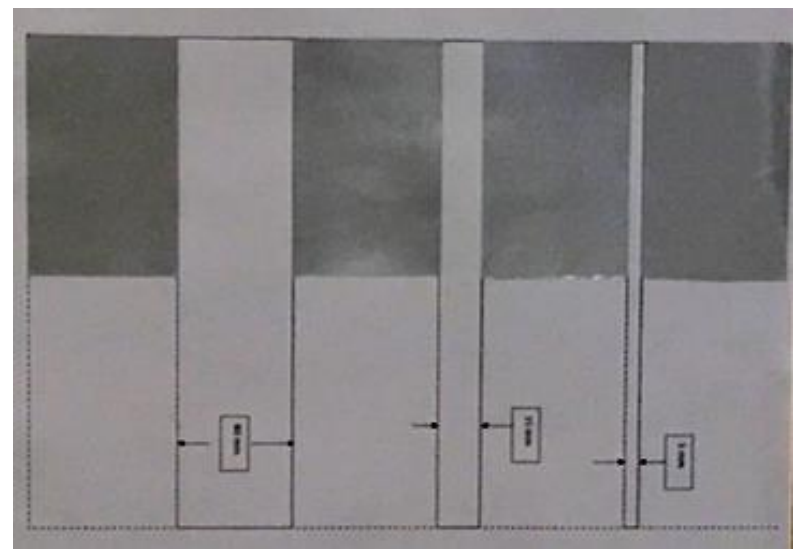

Figure 2 Set-up to measure accuracy, precision and resolution

Scanning was performed at distances of $1,2.5,5$ and $10 \mathrm{~m}$. The resulting point clouds were colourised with respect to reflected light intensity with CloudCompare software (Girardeau-Montaut 2016). In this way, the reflected tapes appeared in red in the visualisation window of CloudCompare. It should be noted that measurements were done not only of these tapes, but also of other structures captured in the scans. Figure 3 shows the point cloud generated at a distance of $1 \mathrm{~m}$ from the board. It is apparent that all the tapes can be resolved at this distance. However, the resolution becomes poorer at larger distances. Tests at mine sites have revealed that objects that are as close as $2 \mathrm{~cm}$ from each other can be resolved at a distance of $5 \mathrm{~m}$ from the source (Figures 4 and 5 ).

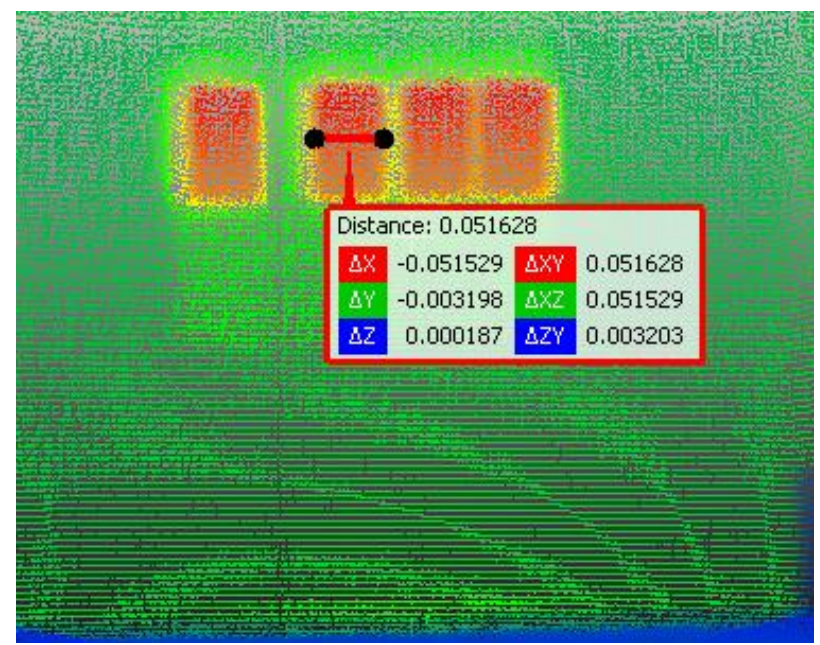

Figure 3 Point cloud at a distance of $1 \mathrm{~m}$ from the board 


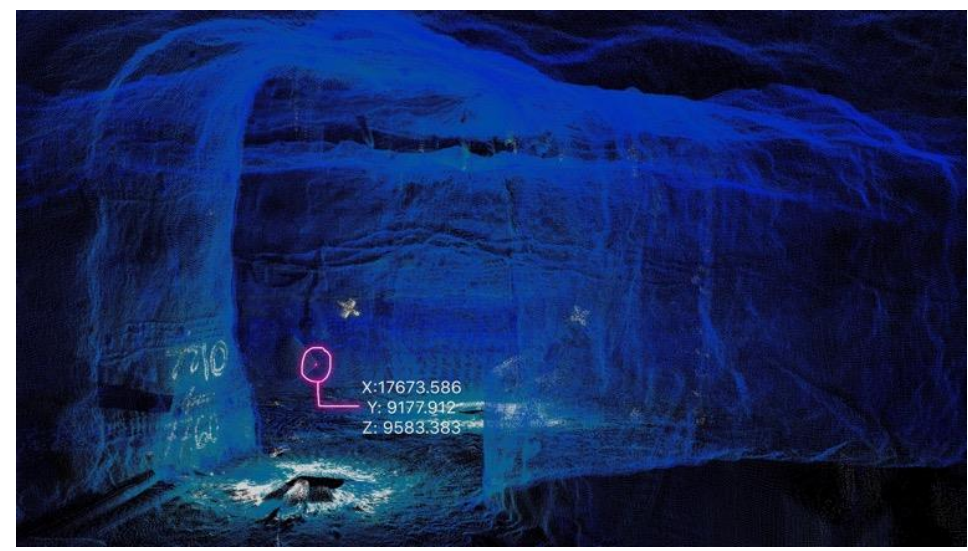

Figure 4 A surveyed point identified in the point cloud captured by V-SCAN3D

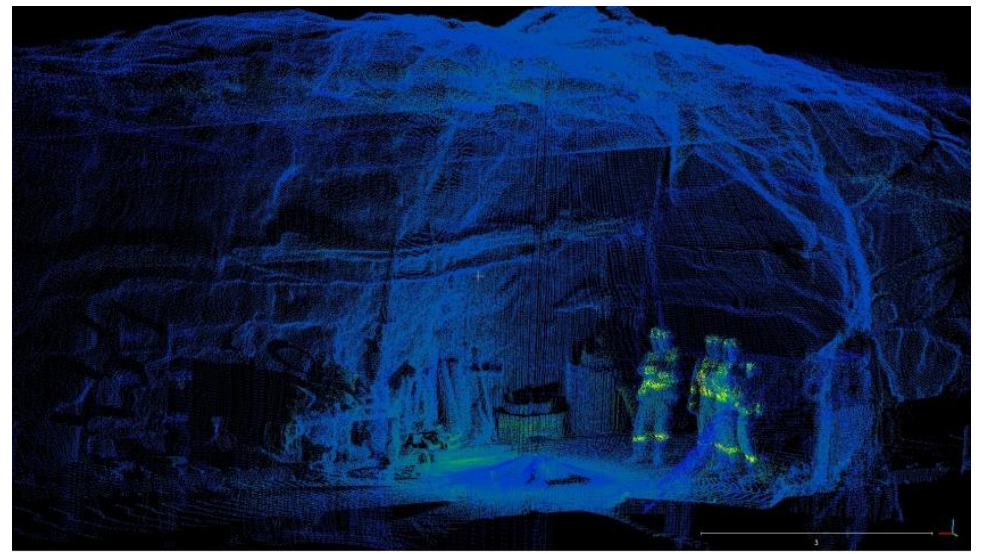

Figure 5 Point cloud of a mine drift captured by V-SCAN3D

Table 1 shows that the system shows a precision in length measurement of about $2 \mathrm{~mm}$ with better than $1 \%$ accuracy at a distance of up to $10 \mathrm{~m}$ from the laser source. This shows a great level of accuracy, since most drifts in underground mines are of the $5 \times 5 \mathrm{~m}$ arch back type, and a control point on the drift wall can be easily identified in the resulting point cloud. This can be seen in Figure 4 as well, where a control point has been identified and circled.

Table 1 Results of measurements

\begin{tabular}{|c|c|c|c|c|c|c|c|c|}
\hline \multirow{2}{*}{$\begin{array}{l}\text { Distance } \\
\text { (m) }\end{array}$} & \multirow{2}{*}{$\begin{array}{l}\text { Actual } \\
\text { (mm) }\end{array}$} & \multicolumn{3}{|c|}{ Measurements (mm) } & \multirow{2}{*}{$\begin{array}{l}\text { Mean } \\
(\mathrm{mm})\end{array}$} & \multirow{2}{*}{$\begin{array}{l}\text { Std. dev. } \\
(\mathrm{mm})\end{array}$} & \multirow{2}{*}{$\begin{array}{l}\text { Precision } \\
(\mathrm{mm})\end{array}$} & \multirow{2}{*}{$\begin{array}{l}\text { Accuracy } \\
(\%)\end{array}$} \\
\hline & & 1 & 2 & 3 & & & & \\
\hline 1 & 51 & 51.6 & 50.2 & 51.8 & 51.2 & 0.87 & 0.50 & 0.4 \\
\hline 2.5 & 264 & 261.7 & 267 & 267.7 & 265.5 & 3.28 & 1.89 & 0.6 \\
\hline 5 & 908 & 905.7 & 909 & 901.6 & 905.4 & 3.71 & 2.14 & 0.3 \\
\hline 10 & 264 & 262.7 & 270.6 & 266.2 & 266.5 & 3.96 & 2.29 & 0.9 \\
\hline
\end{tabular}

Figure 5 shows the point cloud generated with V-SCAN3D at a mine site. The details in the point cloud can be used by geologists to identify rock conditions such as large cracks and faults. This point cloud was generated in less than one minute.

An investigation into if V-SCAN3D can be used to perform stationary scans of a stope was also conducted. In some cases, the stopes are small enough so that the scanner can be inserted into the cavity with a post or a cable. This method is similar to how scanning is done by traditional CMS equipment. Figure 6 shows the scan of a stope by inserting the scanner with a post into the cavity. 


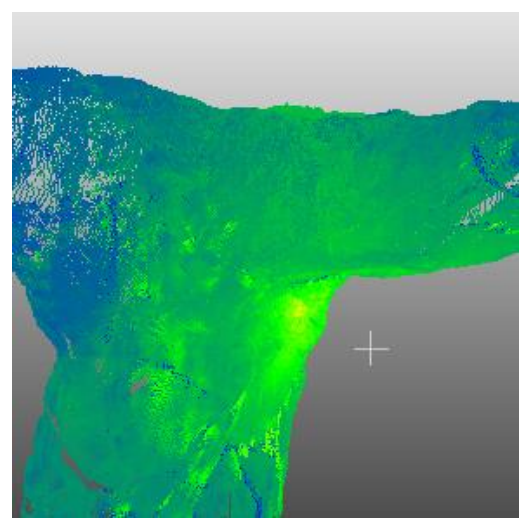

Figure 6 Point cloud of an underground mine stope captured by V-SCAN3D

Next, the use of the system to map mine drifts by performing multiple stationary scans and manually stitching them together was investigated. Figure 7 shows a mine drift scanned in this way with the scanner on the MineFly drone. The drone was flown and landed at certain distances from the operator and stationary scans performed. It should be noted that a part of the drift was inaccessible and traditional CMS from the entry point would have most probably missed the parts circled in the figure.

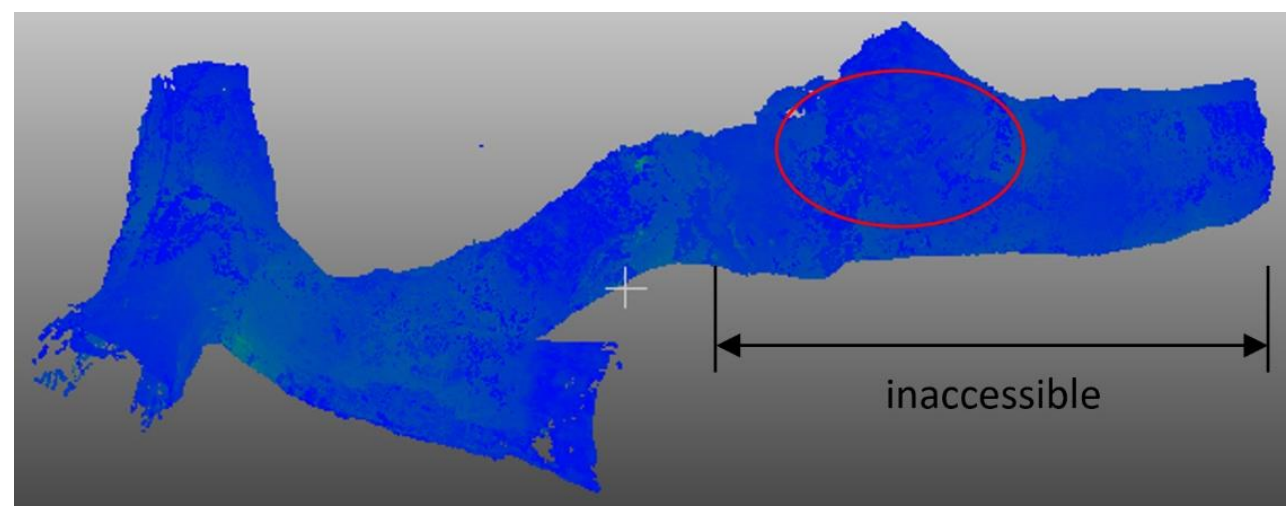

Figure 7 Multiple stationary scans merged together during post processing

\subsection{Mobile scanning}

In many situations, the areas to be scanned and mapped are not only inaccessible, but also too large to perform stationary scans. In these cases, the use of traditional CMS may introduce shadows as the system is kept at one position during scanning. In such situations, mobile scanning can be performed using the SLAM algorithm. To test this, the V-SCAN3D with SLAM capability was used to perform mobile scans in different underground mines. For this, the scanner was mounted on mine ground vehicles and two different drones - TILT Ranger and MineFly.

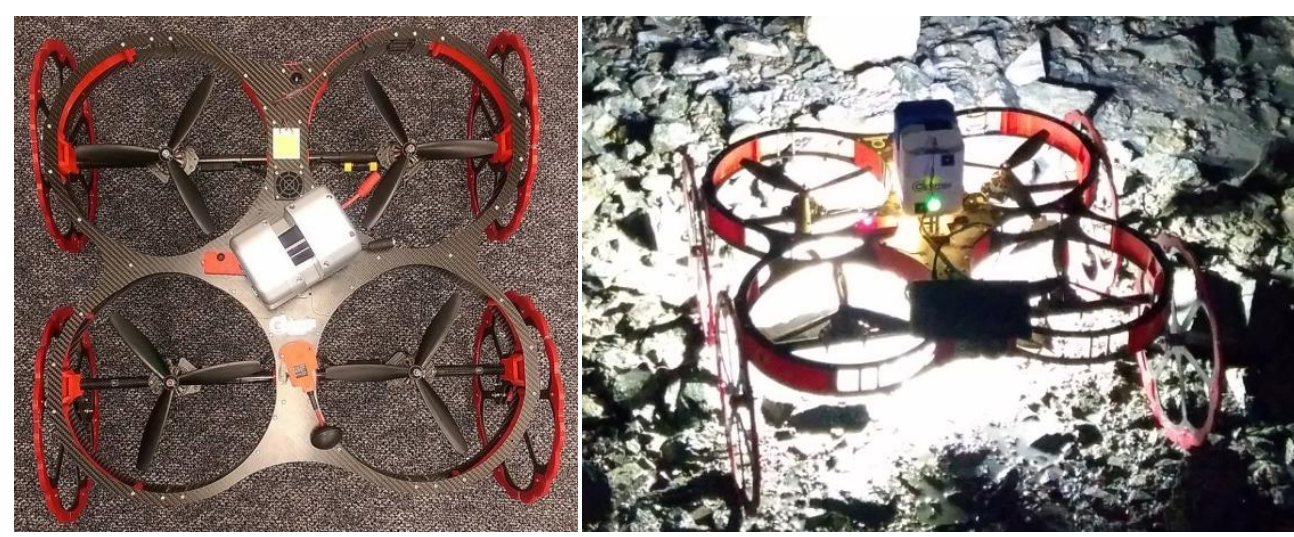

Figure 8 V-SCAN3D mounted on the TILT Ranger drone developed by Inkonova, Sweden 
For the tests with the scanner mounted on a ground vehicle, the real-time version of the scanner, VM-SCAN3D, was used. This system generates the point cloud in real time using the SLAM algorithm. The result of one of the tests at an underground mine is shown in Figure 9. The vehicle was continuously moved between the drifts with the scanner running. The point cloud was then downloaded from the system at the end of the scans and used to generate wire mesh as shown in the figure.

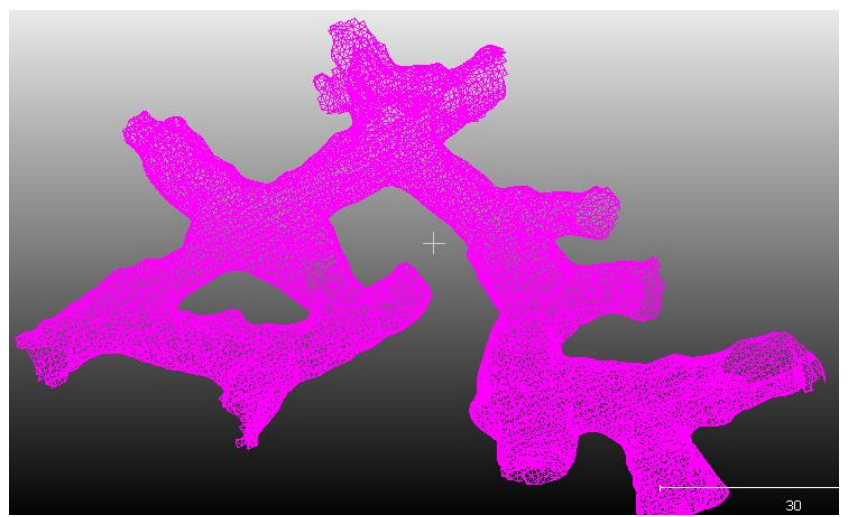

Figure 9 Wire frame of a mobile scan performed by VM-SCAN3D (real-time version) mounted on an underground mine vehicle

Figure 10 shows the wire frames of a stope scanned with traditional CMS equipment (Teledyne Optech Incorporated 2017) and V-SCAN3D mounted on the TILT Ranger drone. The drone-based mobile scanning took less than two minutes and involved flying the drone in and out of the stope with the scanner running in SLAM mode. It is obvious that the scan performed by V-SCAN3D is more detailed than the one performed by the traditional CMS scanner.

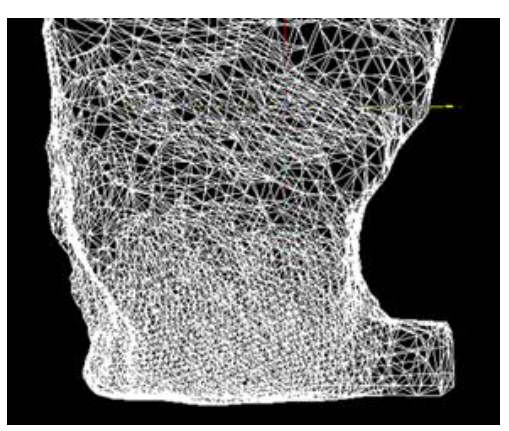

(a)

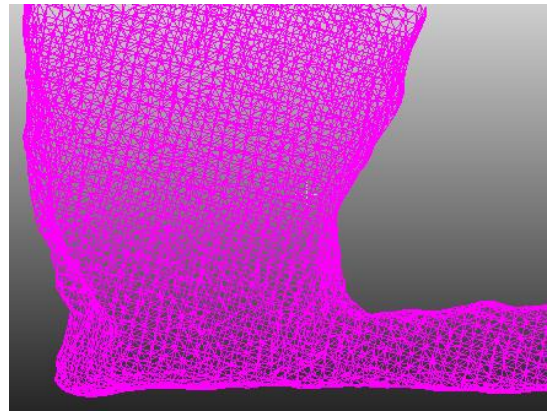

(b)

Figure 10 Wire frame of scan performed by (a) traditional CMS equipment; and, (b) By V-SCAN3D mounted on a TILT Ranger drone

Figure 11 shows the wire frame of a large stope scanned with V-SCAN3D mounted on a TILT Ranger drone. The drone was flown in and out of the stope with the scanner running in SLAM mode.

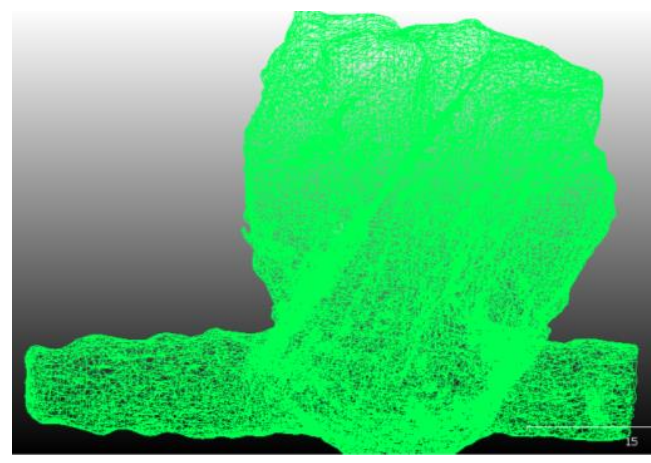

Figure 11 Wire frame of a large stope scanned by V-SCAN3D mounted on a TILT Ranger drone 
It should be pointed out that the MineFly drone has an optional collision avoidance system. However, the guards around both the MineFly and TILT Ranger drones are capable of saving the propellers during low-impact collisions with walls. The TILT Ranger drone has a longer operational range and could also be flown in non-line-of-sight situations during these tests.

\section{Conclusion}

The tests performed in this study validate the hypothesis that fast, lightweight and compact 3D laser scanners having stationary and mobile scanning capabilities can be more effectively used to scan underground mine cavities as compared to traditional CMS scanning equipment. Such systems allow more robust, accurate, quick and safe scanning and mapping of stopes, ore passes, drifts and other underground mine openings. Mobile scanning systems based on ground and aerial vehicles are highly effective in scanning and mapping hard-to-access and inaccessible areas in underground mines and do not suffer from shadowing effects. The high point cloud density obtained from such systems can also be helpful in deducing geological information about rock surfaces.

\section{Acknowledgement}

The authors thank their partners and developers of the TILT Ranger drone, Inkonova, for participating in tests with the TILT Ranger drone. The opportunities to perform various tests provided by NORCAT mine, Vale's Coleman Mine, Glencore's Kidd Mine, Randgold's Loulo mine and Glencore's Nickel Rim South mine are acknowledged and greatly appreciated.

\section{References}

Amann, M-C, Bosch, T, Myllyla, R \& Rioux, M 2001, 'Laser ranging: a critical review of usual techniques', Optical Engineering, vol. 40, no. 1, pp. 10-19.

Girardeau-Montaut, D 2016 CloudCompare, viewed 30 June 2017, http://www.cloudcompare.org/

Teledyne Optech Incorporated 2017, CMS V500 Cavity Monitoring System, viewed 30 June 2017, http://www.teledyneoptech.com 
